\title{
Efectividad de la consejería en enfermería en la adherencia al tratamiento farmacológico en pacientes con tuberculosis de la micro red de salud Jaime Zubieta Calderón en San Juan de Lurigancho, Lima, Perú
}

\author{
Andrea Castañeda-Navarrete 1,a, Dunia Sánchez- León a, Milagros Manchego- Quispe ${ }^{2, a}$, \\ Yesenia Musayón- Oblitas ${ }^{3, b, c}$.
}

\section{RESUMEN}

Objetivos: determinar la efectividad de la consejería en enfermería en la adherencia al tratamiento farmacológico en pacientes nuevos con tuberculosis sensible al tratamiento, en la Micro red de salud Jaime Zubieta Calderón perteneciente a la DISA IV de Lima Este en San Juan de Lurigancho durante los meses de octubre 2013 hasta agosto 2014. Material y Métodos: estudio cuasi experimental con grupo control histórico. La muestra estuvo conformada por 30 pacientes nuevos con diagnóstico tuberculosis sensible, los controles se tomaron teniendo los mismos criterios de inclusión y el periodo de tiempo durante los meses de octubre 2012-setiembre 2013 obteniendo 72 controles. La recolección de datos se realizó a través de la tarjeta de control de asistencia y administración de medicamentos de cada uno. Los resultados se tabularon en el programa Excel 2010, posteriormente analizados en el programa SPSS versión 22. Resultados: se realizó un cuadro de homogeneidad donde se evidenció un nivel de significancia estadística 4.62, siendo el valor de $\mathrm{p}=0.032$. El grupo intervenido obtuvo $100 \%$ de adherencia; a diferencia del grupo control histórico que tuvo una adherencia $86 \%$, se evidenció un incremento del $14 \%$ a lo esperado. Conclusiones: La consejería de enfermería es efectiva en la mejora de la adherencia al tratamiento antituberculoso en pacientes con TB sensible. Además, favorece a la asistencia diaria a la toma del tratamiento, citas médicas, nutrición y enfermería.

PALABRAS CLAVE: adherencia, tuberculosis sensible, consejería en enfermería.

\section{Effectiveness of the counseling in nursing adherence to drug treatment in patients with tuberculosis of the micro red de salud Jaime Zubieta Calderón in San Juan de Lurigancho, Lima, Perú}

\section{SUMMARY}

Objectives: to determine the effectiveness of counseling in nursing adherence to drug treatment in new patients with tuberculosis in "Micro red de salud Jaime Zubieta Calderon" belongs to the DISA IV Lima Este in San Juan de Lurigancho district during the months of October 2013 to August 2014. Material and Methods: a quasi-experimental research design with historical control. The sample was conducted with 30 patients new sensitive diagnosed with tuberculosis, where evaluated over a period of two months-a period greater risk to non-adherence- issues about the disease, treatment is addressed, and the particular needs of each user is identified. Controls were taken to have the

Laboratorio Farmindustria. Lima, Perú.

Clínica Ricardo Palma. Lima, Perú.

Facultad de Enfermería, Universidad Peruana Cayetano Heredia. Lima, Perú.

Licenciada en enfermería ; b Doctora en Salud Pública ; c Profesora Principal 
same inclusion criteria and the period during the months of October 2012 to September 2013 obtaining 72 controls. The results are tabulated in the Excel 2010 program subsequently analyzed in SPSS version 22. Results: Homogeneity box where a level of statistical significance was performed evidenced 4.62, the value of $p=0.032$. The interview group the percentage of adherence was 100\%; than the history control group had a $86 \%$ adherence is achieved a $14 \%$ increased the percentage expected. Conclusions: Nursing counseling is effective in improving adherence to tuberculosis treatment in patients with sensitive TB. Furthermore, fauvors the daily attendance taking treatment, medical appointments, nutrition and nursing.

KEYWORDS: Adherence, sensitive tuberculosis, nursing counseling.

\section{INTRODUCCIÓN}

La Organización Mundial de la Salud (OMS) declaró la tuberculosis como una urgencia mundial agravada, cerca de la tercera parte de la población mundial está infectada con el Mycobacterium (1). E1 95\% de casos y el 98\% de las muertes ocurrieron en países en vía de desarrollo, las muertes por TBC corresponden al $25 \%$ de la mortalidad evitable. En América del Sur, el mayor número de casos y las tasas más altas de incidencia se concentran en Brasil y Perú (2).

La tuberculosis es un problema de salud pública de gran relevancia y es la principal causa de muerte por enfermedades infecciosas en personas adultas. Según cifras oficiales de la Estrategia Sanitaria Nacional de Prevención y Control de la Tuberculosis en el año 2006, cada día fallecen tres personas por esta enfermedad y cada hora 4 personas son diagnosticadas de tuberculosis (3). Sin embargo, según datos oficiales de la Organización Mundial de Salud (OMS), considerando el porcentaje de detección del programa y estimaciones del sub-registro para ese mismo año, el número de enfermos se incrementa a seis por hora en el Perú (4).

La Organización Mundial de la Salud ha estimado que de no emprender medidas de control adicionales, para el año 2020, el número de casos nuevos se habrá elevado a 10 millones (2). En el Perú, la tasa de abandono al tratamiento corresponde a un $5,8 \%$, cifra que rebasa el límite que establece el programa de PCT (5\% de abandono) (5). La adherencia puede medirse utilizando cualquiera de los dos procesos orientados hacia la obtención de resultados o definiciones al término del tratamiento (6).

Los factores de riesgo de no adherencia al tratamiento son diversos, entre ellos se encuentran el estigma social de la enfermedad, las reacciones adversas, la intolerancia a los medicamentos y la relación no asertiva entre el personal de salud y paciente, estos han sido ampliamente investigados sobre todo en los países desarrollados. Se requiere realizar investigación epidemiológica del fenómeno con el fin de conocer los factores específicos en cada área. Probar mediante estudios experimentales estrategias de mejoramiento de adherencia con intervenciones a nivel individual y colectivo (7).

Existen métodos para determinar la adherencia de un paciente al tratamiento como el registro de la asistencia diaria ESN - PCT donde se suministran los medicamentos, reporte de paciente, conteo de tabletas. Por el contrario, la sospecha de no adherencia se da cuando el paciente no asiste a la toma de su medicamento por dos días consecutivos y se considera abandono a la inasistencia de la persona al tratamiento por más de 30 días (3).

La consejería puede ser aplicada en nuestro medio para disminuir el abandono al tratamiento en pacientes con tuberculosis implementándose una "cadena educacional" mediante la cual se entrene al personal de salud en la importancia del problema y sus estrategias de abordaje y éste a su vez eduquen a las personas afectadas (8).

Un factor clave para lograr que el paciente acuda a recibir su terapia farmacológica es la información y consejería que se brinde al momento de la entrevista de enfermería o cada vez que el paciente acuda a sus controles (9). La Consejería es una herramienta de comunicación por excelencia, una relación de ayuda que pretende implicar al individuo, a partir de sus propias necesidades y emociones. Mediante la Consejería se brinda orientación, información, apoyo emocional y se ayuda a tomar decisiones $(10,11)$. la define a la Consejería como "un diálogo confidencial entre el cliente y el consejero con el propósito de que el cliente logre lidiar y contribuir con el mejoramiento de su salud de manera que pueda tomar decisiones personales relacionadas con su enfermedad" (12).

La Norma Técnica de Salud para el Control de la Tuberculosis plantea que la organización de la atención integral e individualizada de la persona con Tuberculosis y Tuberculosis multidrogorresistente, su familia y 
la comunidad, es responsabilidad del profesional de enfermería, por ello como consejero desarrolla un papel preponderante en la toma de decisiones del aconsejado y en su orientación para la solución de sus problemas (13). La falta de información básica sobre la enfermedad, lleva a los pacientes a tomar alternativas, entre ellas, asistir o no al tratamiento antituberculoso. Es ahí donde la enfermera tiene el papel preponderante como agente de salud, pues es la más próxima a la persona y es un canal de información directa, ella es mediadora de conflictos, puesto que sus cuidados se dirigen y se enfocan en las dificultades, obstáculos y problemas, de los requirentes de esa atención. Con la finalidad de sensibilizar y capacitar, para el fortalecimiento de su autonomía con respecto a su enfermedad, y ofrecer ayuda profesional en forma eficaz y eficiente (14).

La pobre adherencia al tratamiento constituye el principal obstáculo para el control global de la enfermedad; por ello, es importante articular el programa de control de la TBC con otras estancias como la economía, la política, la ética y el quehacer propio de las organizaciones que velan por el bienestar de los pueblos; para convertir las acciones en un frente común de prevención y control de la TBC (13).

El objetivo de la presente investigación fue determinar ¿Cuál es la efectividad de consejería de enfermería para mejorar la tasa de adherencia al tratamiento farmacológico en pacientes con tuberculosis en el centro de salud Jaime Zubieta Calderón en San Juan de Lurigancho, Lima, Perú?

\section{MATERIAL Y METODOS}

El estudio fue cuasi experimental con control histórico con mediciones antes y después de la consejería, realizado en la micro red de salud Jaime Zubieta Calderón perteneciente a la DISA IV Lima Este, ubicado en el distrito de San Juan de Lurigancho. La muestra estuvo conformada por 30 pacientes con diagnostico de tuberculosis sensible que se encuentren en la primera fase de tratamiento, el grupo control histórico estuvo conformado por 72 pacientes que recibieron el tratamiento antituberculoso durante octubre 2012 hasta setiembre 2013.

La recolección de datos se realizó a través de la tarjeta de control de asistencia, administración de medicamentos y la "Guía para las buenas prácticas de consejería en tuberculosis" del Ministerio de Salud-2006, modificado por las autoras y validado por diez expertos con conocimiento del tema. La guía describe la actitud que el personal de enfermería debe adoptar al iniciar la sesión (consideraciones específicas, funciones del consejero, metodología y recomendaciones). Especifica preguntas que el consejero debe realizar al paciente con el fin de valorar el nivel de conocimiento respecto a la enfermedad, describe las respuestas de manera clara y específica correspondientes a las preguntas realizadas al paciente.

El proyecto de investigación fue aprobado por el Comité Institucional de Ética de la Universidad Peruana Cayetano Heredia. El proceso de recolección de datos se realizó durante los meses de octubre del 2013 hasta agosto del 2014.

Se inició con la entrega de la guía de consejería de enfermería a la enfermera encargada de la ESN-PCT de la micro red de salud Jaime Zubieta, quien cumplía con el perfil del consejero y conocía las fases de la consejería. Antes de aplicar la consejería de enfermería se aplicó al paciente la finalidad del estudio, obteniendo su consentimiento. Luego se procedió con el desarrollo de la consejería. Se registraron el seguimiento de toma del tratamiento, asistencia a la cita médica, de enfermería y nutricional, entrega mensual de muestra de esputo, peso al inicio del tratamiento, al mes y segundo mes mediante la tarjeta de control de asistencia y administración de medicamentos con esquema uno de la ESN - PCT.

Para la recolección de datos del control histórico, se identificó a través de las historias clínicas a los pacientes que asistieron al ESN-PCT durante el periodo Octubre 2012 hasta setiembre 2013 que cumplieran con los criterios de inclusión.

Una vez recolectado los datos, se introdujeron los resultados de la guía de identificación en una base de datos creada en el programa Excel 2010, posteriormente analizados en el programa SPSS versión 22., identificando proporciones para cada una de las variables estudiadas según algunas características de la muestra.

Se aplicó el análisis bivariado entre la adherencia al tratamiento antituberculoso y la aplicación de consejería de enfermería. El análisis incluyó la aplicación de Chi cuadrado para evaluar asociación entre ambas variables, determinando la significancia estadística en la adherencia al tratamiento antituberculoso antes y después de la consejería.

\section{RESULTADOS}

Se observó que un 86\% (62/72) del grupo control histórico se adhirió al tratamiento, mientras que un 100\% (30/30) del grupo intervenido se adhirió al tratamiento antituberculoso 
en la primera fase del esquema. Se utilizó la prueba de Chi- Cuadrado, obteniendo un nivel de significancia de 4,$62 ; \mathrm{p}=0,032($ tabla 1$)$.

Con respecto a la adherencia al tratamiento antituberculoso del grupo control histórico del centro de salud Jaime Zubieta Calderón, se observa que un 86\% (62/72) se adhirió al tratamiento, mientras que un 14\% (10/72) no se adhirió satisfactoriamente (tabla 2).

Se observó que el grupo intervenido después de la consejería en enfermería se adhiere al tratamiento en un $100 \%(30 / 30)$ en la primera fase del esquema (tabla 3$)$.

Al comparar la asistencia al tratamiento farmacológico de ambos grupos se observa que el grupo intervenido tiene un
$10 \%$ de más asistencia que el grupo control histórico tanto en el primer mes como en el segundo mes. Al comparar semana por semana la asistencia siempre fue mayor en el grupo intervenido (tabla 4).

Se observa que un 93\% del grupo control histórico asistió a sus citas de enfermería, mientras que en el grupo intervenido la asistencia fue de un $100 \%$, siendo esta diferencia estadísticamente significativa $(\mathrm{p}=0.014)($ tabla 5$)$.

Se observó que un $64 \%$ del grupo control histórico asistió a sus citas programadas de nutrición a diferencia del grupo intervenido que asistió en un $83 \%$, siendo esta diferencia estadísticamente significativa $(\mathrm{p}=0.052)($ tabla 6).

Tabla 1.Tasa de adherencia al tratamiento del grupo control histórico y del grupo intervenido después de la consejería en enfermería del Centro de Salud Jaime Zubieta

Calderón.

ADHERENCIA AL TRATAMIENTO

\begin{tabular}{cccccc} 
Grupo de Estudio & $\begin{array}{c}\text { Muestra } \\
\mathbf{N}\end{array}$ & $\begin{array}{c}\text { Adheridos } \\
\mathbf{N}\end{array}$ & $\mathbf{\%}$ & $\mathbf{X 2}$ & $\mathbf{p}$ \\
\hline Control Histórico & 72 & 62 & 86 & & \\
(Oct.2012 - Set.2013) & & & 4,62 & 0,032 \\
Grupo Intervenido & 30 & 30 & 100 & \\
$($ Oct.2013 - Ago.2014) & & & & \\
\hline
\end{tabular}

Tabla 2. Adherencia al tratamiento del grupo control histórico del Centro de Salud Jaime Zubieta Calderón.

\begin{tabular}{ccc}
\hline ADHERENCIA AL TRATAMIENTO & \multicolumn{2}{c}{$\begin{array}{c}\text { CONTROL HISTÓRICO } \\
\text { (Oct. 2012 - Set. 2013) }\end{array}$} \\
\hline SI & N & 86 \\
NO & 62 & 14 \\
Total & 10 & $100 \%$ \\
\hline
\end{tabular}

Tabla 3. Tasa de adherencia al tratamiento del grupo intervenido después de la consejería del Centro de Salud Jaime Zubieta Calderón.

\begin{tabular}{ccc}
\hline & \multicolumn{2}{c}{ GRUPO INTERVENIDO } \\
ADHERENCIA AL TRATAMIENTO & (Oct. 2013 - Ago. 2014) \\
& N & $\%$ \\
\hline SI & 30 & 100 \\
NO & 0 & 0 \\
Total & 30 & $100 \%$ \\
\hline
\end{tabular}


Tabla 4. Comparación de la asistencia al tratamiento farmacológico del grupo control histórico y grupo intervenido con la consejería de enfermería en el Centro de Salud Jaime Zubieta Calderón.

\begin{tabular}{|c|c|c|c|c|c|c|}
\hline \multirow{2}{*}{$\begin{array}{c}\text { MES } \\
\mathbf{1}^{\circ} \mathrm{mes}\end{array}$} & \multicolumn{2}{|c|}{$\begin{array}{c}\text { CONTROL } \\
\text { HISTÓRICO } \\
\text { (Oct. 2012 - Set. 2013) }\end{array}$} & \multicolumn{2}{|c|}{$\begin{array}{l}\text { GRUPO INTERVENIDO } \\
\text { (Oct. } 2013 \text { - Ago. 2014) }\end{array}$} & \multirow[t]{2}{*}{$\mathbf{X} 2$} & \multirow[t]{2}{*}{$\mathbf{p}$} \\
\hline & $\mathbf{N}$ & $\%$ & $\mathbf{N}$ & $\%$ & & \\
\hline 1ra semana & 62 & 86,1 & 30 & 100 & 4,62 & 0,032 \\
\hline 2da semana & 65 & 90,3 & 29 & 96,7 & 1,96 & 0,274 \\
\hline 3ra semana & 60 & 83,3 & 28 & 93,3 & 1,79 & 0,181 \\
\hline 4ta semana & 63 & 87,5 & 29 & 96,7 & 2,01 & 0,156 \\
\hline Promedio & \multicolumn{2}{|c|}{87} & \multicolumn{2}{|c|}{97} & & \\
\hline $2^{\circ}$ mes & $\mathbf{N}$ & $\%$ & $\mathbf{N}$ & $\%$ & $\mathbf{X} 2$ & $\mathbf{P}$ \\
\hline 1ra semana & 61 & 84,7 & 29 & 96,7 & 2,91 & 0,088 \\
\hline 2da semana & 62 & 86,1 & 28 & 93,3 & 1,06 & 0,302 \\
\hline 3ra semana & 61 & 84,7 & 30 & 100 & 5,14 & 0,023 \\
\hline 4ta semana & 70 & 97,2 & 30 & 100 & 0,85 & 0,053 \\
\hline Promedio & \multicolumn{2}{|c|}{88} & \multicolumn{2}{|c|}{98} & & \\
\hline
\end{tabular}

Tabla 5.Asistencia a citas de enfermería del grupo control histórico y el grupo intervenido después de la consejería en el Centro de Salud Jaime Zubieta Calderón.

\begin{tabular}{ccccccr}
\hline CUMPLIMIENTO & \multicolumn{2}{c}{ CONTROL HISTÓRICO } & \multicolumn{2}{c}{ GRUPO INTERVENIDO } \\
DE CITAS DE & (Oct. 2012 - Set. 2013) & Oct. 2013- Ago. 2014) & X2 & p \\
ENFERMERÍA & $\mathbf{N}$ & $\mathbf{\%}$ & $\mathbf{N}$ & $\mathbf{\%}$ & & \\
\hline SI & 67 & 93 & 30 & 100 & & \\
NO & 5 & 7 & 0 & 0 & 2,19 & 0,014 \\
\hline
\end{tabular}

Tabla 6. Asistencia a citas de nutrición del grupo control histórico y el grupo intervenido después de la consejería de enfermería en el Centro de Salud Jaime Zubieta Calderón.

\begin{tabular}{|c|c|c|c|c|c|c|}
\hline \multirow{2}{*}{$\begin{array}{l}\text { CUMPLIMIENTO } \\
\text { DE CITAS DE } \\
\text { NUTRICIÓN }\end{array}$} & \multicolumn{2}{|c|}{$\begin{array}{c}\text { CONTROL } \\
\text { HISTÓRICO } \\
\text { (Oct. 2012 - Set. 2013) }\end{array}$} & \multicolumn{2}{|c|}{$\begin{array}{c}\text { GRUPO } \\
\text { INTERVENIDO } \\
\text { (Oct. 2013 - Ago. 2014) }\end{array}$} & \multirow[t]{2}{*}{$\mathrm{X} 2$} & \multirow[t]{2}{*}{ p } \\
\hline & $\mathbf{N}$ & $\%$ & $\mathbf{N}$ & $\%$ & & \\
\hline SI & 46 & 64 & 25 & 83 & \multirow{2}{*}{3,78} & \multirow{2}{*}{0,052} \\
\hline NO & 26 & 36 & 5 & 17 & & \\
\hline
\end{tabular}

\section{DISCUSIÓN}

Se han realizado diversas intervenciones buscando la manera más adecuada para mejorar la adherencia al tratamiento, la tuberculosis es una enfermedad transmisible, por lo tanto la adherencia deficiente a un tratamiento prescrito aumenta los riesgos de farmacorresistencia, morbilidad y mortalidad, en el orden individual y comunitario. La adherencia deficiente a la medicación antituberculosa, es una barrera principal para el control mundial (5).
Los resultados obtenidos en nuestro estudio confirman que la consejería en enfermería contribuye de manera directa a lograr la adherencia al tratamiento antituberculoso y culminar con éxito, dado que, los resultados son significativos estadísticamente $(\mathrm{p}=0,032)$.

Numerosos estudios han abordado desde diferentes perspectivas la problemática de la no adherencia, en un estudio realizado por Morisky informó que con un programa educativo había aumentado la adherencia de $38 \%$ a $68 \%(15)$. 
Por otro lado en nuestro país, Arriola y col., con el objetivo de determinar algunos factores asociados a la asistencia al tratamiento antituberculoso realizaron un estudio de diseño descriptivo transversal en el Programa de Control de Tuberculosis (PCT) en el hospital de Puente Piedra y en el Centro Materno Infantil Zapallal de Puente Piedra, en el cual la población estuvo conformada por 77 pacientes con diagnóstico de tuberculosis pulmonar. Los datos obtenidos en los resultados del total de pacientes fueron que el 39\% presentó asistencia discontinua al PCT y entre los factores asociados en la asistencia discontinua con mayor significancia fue el no tener la información básica de la enfermedad (16).

Por su lado Tacuri realizó una investigación sobre "Relación entre la adherencia y el nivel de conocimiento sobre el tratamiento de los pacientes con tuberculosis en el Centro de Salud Primavera" en El Agustino - Lima,Perú 2009, con el objetivo de determinar la relación entre adherencia y nivel de conocimiento sobre el tratamiento de los pacientes con tuberculosis. Utilizó el método descriptivo correlacional de corte transversal, la muestra estuvo constituída por 38 pacientes con diagnóstico de tuberculosis pulmonar registrados en la ESN-PCT del Centro de Salud Primavera. Las conclusiones fueron que existe relación directa entre adherencia y el nivel de conocimiento de los pacientes con tuberculosis; es decir, que la no adherencia estuvo presente en los pacientes que tienen el nivel conocimientos de medio a bajo y quienes son adherentes al tratamiento tienen un nivel alto de conocimiento resultado obtenido mediante la prueba de Chi cuadrado con un nivel de significancia de $95 \%$ de confianza (17).

Los anteriores estudios fueron citados con el fin de resaltar que la consejería como estrategia es fundamental para ofrecer elementos importantes, entre ellos proporcionar la información veraz y suficiente de acuerdo a la necesidad y conocimientos del usuario.

La consejería tiene como objetivo educar al paciente, brindando soporte al manejo integral que permita eliminar ideas erróneas que puedan confundir al paciente, haciéndole comprender el problema que enfrentan y la importancia de la adherencia al tratamiento (14).

La Norma Técnica de Tuberculosis considera sospecha de no adherencia cuando se evidencia que el paciente no asiste a la toma de sus medicamentos por dos días consecutivos y se considera abandono cuando el paciente registra inasistencia por más de 30 días. La intervención se aplicó en la primera fase de tratamiento, el porcentaje en cuanto al cumplimento en el primer mes y segundo mes de tratamiento del grupo control histórico es inferior en relación al grupo intervenido donde el cumplimiento incrementó en un 10\% en ambos meses (1er mes tratamiento: control histórico: 87\% vs. grupo intervenido: 97\%; 2do mes tratamiento: control histórico: $88 \%$ vs. grupo intervenido: $98 \%$ ). Cabe resaltar que no se registró faltas por más de dos días consecutivos, es así que la intervención de enfermería basada en consejería, influye en el cumplimiento del tratamiento farmacológico, en relación al grupo control histórico.

Con los resultados expuestos, se concuerda con lo recomendado en la investigación titulada "acción de salud pública" de Hoa y col., donde mencionan que las intervenciones basadas en mejorar la adherencia del tratamiento antituberculoso debe ser aplicado en el primer periodo de tratamiento (14). Según los resultados encontrados en el estudio realizado por las autoras evidencia que hay mayor riesgo de abandono durante el primer periodo de tratamiento.

Además, se observa que con respecto a las citas programadas en enfermería y nutrición que se controla mediante la tarjeta de control de asistencia y administración de medicamentos con esquema uno, favorece en un 7\% (control histórico: 93\% vs grupo intervenido $100 \%$ ) con una significancia estadística $\mathrm{p}=0,014$ y $19 \%$ (control histórico: $64 \%$ vs. grupo intervenido: $83 \%$ ) significancia estadística $\mathrm{p}=0,05$ respectivamente.

Los resultados encontrados demuestran que existe mayor adherencia al tratamiento en el grupo intervenido que en el grupo control histórico, incrementando más de lo esperado. Dentro de esto, el grupo control histórico mantuvo una adherencia al tratamiento en un $86 \%$, mientras que el grupo intervenido al cual se aplicó la consejería de enfermería logro obtener el $100 \%$. Inicialmente se consideró un incremento de adherencia en un 5\%, pero se superó en un 14\% a lo esperado, demostrando que la consejería de enfermería fue efectiva.

La consejería se ha hecho sinónimo de asesoría y consultoría e independiente de cómo se le denomine, implica una relación para brindar orientación, información, contención, apoyo, evaluación y discusión de estrategias de acción para mantener una situación favorable.

El consejero debe dirigir la sesión de forma sutil, buscando conocer la demanda real del usuario, favoreciendo un ambiente que facilite el diálogo entre ambos. Es necesario evitar enjuiciar, etiquetar y regañar a la persona, lo que permitirá crear una atmósfera de cordialidad, entendimiento y respeto y conservar la confidencialidad (12). 
En la intervención el consejero (la Licenciada en enfermería encargada de ESN - PCT) tuvo que seguir ciertas pautas determinadas en la "Guía para las buenas prácticas de consejería en tuberculosis"; la cual, fue explicada en una capacitación previa, se verificó que el consejero cumpliera con todas estas pautas durante la capacitación brindada, simulando una consejería para poder identificar cuáles eran sus debilidades y actuar en ellas.

Se plantearon diversos tipos de casos de pacientes para poder desarrollar el área empática del consejero. Es importante que el consejero este altamente motivado, se sienta identificado y comprometido en la problemática; asimismo, disposición para participar en programas de seguimiento, evaluación y continuidad de las actividades ejecutadas, para la mejora constante del servicio. Las características y habilidades del consejero son importantes para establecer empatía, transmitir calidez y mostrar sensibilidad con el paciente (11).

Es importante aplicar la consejería de manera adecuada durante todo el proceso del tratamiento para la mejora a la adherencia al tratamiento antituberculoso, evitando así el aumento de la morbimortalidad, la probabilidad de contagio a otras personas, aparición de la resistencia bacteriana y el aumento de costo del tratamiento antituberculoso.

\section{REFERENCIAS BIBLIOGRAFICAS}

1. World Health Organization. Multidrug and extensively drugresistant TB (M/XDR-TB). 2010 Global Report on Surveillance and Response. Washington: WHO; 2010.

2. Culqui D, Grijalva C, Reategui S, Cajo J, Suárez L. Factores pronósticos del abandono del tratamiento antituberculoso en una región endémica del Perú. Rev Panam Salud Pública. 2005; 18(1):14-20.

3. Ministerio de Salud. Norma Técnica de Salud para el Control de la tuberculosis/Ministerio de salud. Dirección general de salud de las personas. Estrategia sanitaria nacional de prevención y control de tuberculosis. Lima: MINSA; 2006.p. 277.

4. World Health Organization. Global report tuberculosis control, surveillance, planning, financing. Washington: WHO; 2008.

5. Organización Panamericana de la Salud. Sistemas de salud basados en la Atención Primaria de Salud: estrategias para el desarrollo de los equipos de APS. Washington, D.C.: OPS; 2008.

6. Volmink J, Garner P. Interventions for promoting adherence to tuberculosis management. Cochrane Database of Systematic Reviews, 2005.

7. Ribeiro S, Amado V, Camelier A, Fernandes M, Schenkman $\mathrm{S}$. Estudio caso-controle de indicadores de abandono em doentes com tuberculose. J Pneumol. 2004:26(6):291-6.
8. Instituto Nacional de Salud. Diálogo deliberativo del Resumen de Política de Intervenciones dirigidas a disminuir el abandono al tratamiento antituberculoso. Lima: INSUNAGESP; 2011.

9. Centro Nacional de Vigilancia Epidemiológica y Control de Enfermedades. Manual de Procedimientos en Tuberculosis para Personal de Enfermería. Ciudad de Mexico: Centro Nacional de Vigilancia Epidemiológica y Control de Enfermedades; 2007.

10. Organización Panamericana de la Salud. Consejería orientada en los jóvenes para prevenir VIH/ITS y para promover la salud sexual y reproductiva: una guía para proveedores de primera línea. Washington, D.C.: Organización Panamericana de la Salud; 2005. p.184.

11. Subsecretaría de Salud Pública, División de prevención y control de enfermedades, Programa nacional de prevención y control del VIH/SIDA e ITS. Manual de formación básica en consejería para el VIH y las ITS. Santiago: Subsecretaría de Salud Pública;2011.

12. Millan T, Morera I, Vargas N. Consejería a adolescentes: descripción epidemiológica y motivos de consulta. Rev méd Chile. 2007; 135(4):457-463.

13. Ministerio de Salud. Norma Técnica de Salud para el Control de la tuberculosis/Ministerio de salud. Dirección general de salud de las personas. Estrategia sanitaria nacional de prevención y control de tuberculosis. Documento técnico: consejería en tuberculosis, Confección VIH/TB Y TB MDR Lima: MINSA; diciembre 2006.

14. Rodriguez N, Rubio T. Bases conceptuales de la consejería en enfermería. In Crescendo. 2010; 1(2): 319-323.

15. Álvarez-Gordillo G, Álvarez-Gordillo J, Dorantes-Jiménez J, Halperin-Frisch D. Percepciones y prácticas relacionadas con la tuberculosis y la adherencia al tratamiento en Chiapas, México. Salud Publica Mex. 2004; 42:520-52.

16. Arriola-Huerta P, Castillo-Cahuana T, Quispe-Fabián G, Torres-Deza C. Factores asociados a la asistencia del paciente al tratamiento antituberculoso. Rev Enferm Herediana. 2011; 4(2):86-92.

17. Tacuri M. Relación entre adherencia y nivel de conocimientos sobre el tratamiento de los pacientes con tuberculosis en el Centro de Salud Primavera. Tesis para optar el título de Licenciada en Enfermeria. Lima,Perú: Universidad Nacional Mayor de San marcos; 2009.

\section{Correspondencia:}

Flor Yesenia Musayon Oblitas

Correo electrónico: yesenia.musayon@upch.pe

Fecha de Recepción: 03 de febrero del 2016.

Fecha de aceptación: 15 de marzo del 2016. 